\title{
Depolarization of light due to the optical Kerr effect in low-birefringence single-mode fibers
}

\author{
Bruno Crosignani \\ Dipartimento di Fisica, Universitá degli Studi di Roma "La Sapienza," Roma, Italy \\ Benedetto Daino \\ Fondazione Ugo Bordoni, Istituto Superiore Poste e Telecommunicazioni, Roma, Italy \\ Paolo Di Porto \\ Dipartimento di Fisica dell'Università dell'Aquila, L'Aquila, Italy
}

Received December 20, 1985; accepted April 4, 1986

\begin{abstract}
The evolution of the state of polarization of an electromagnetic field propagating in a low-birefringence single-mode fiber in the presence of the nonlinear interaction associated with the optical Kerr effect is investigated. We explicitly evaluate the degree of polarization $P(z)$ as a function of the fiber length $z$ for a completely polarized input field $[P(0)=1]$ possessing an exponential intensity distribution, such as the one furnished by a multimode laser. Furthermore, the asymptotic value $P(\infty)$ corresponding to an arbitrary distribution of the input intensity is determined.
\end{abstract}

\section{INTRODUCTION}

Optical fibers provide a useful tool for studying a number of nonlinear phenomena over propagation distances long enough to furnish relevant effects at relatively low electromagnetic intensities (see, e.g., Refs. 1-4). In this frame, the intensity-induced rotation of the polarization ellipse connected with the opticalKerr effect, which was first investigated for bulk media, ${ }^{5}$ has recently been considered for lowbirefringence single-mode fibers. ${ }^{6}$ In particular, Ref. 6 provides a quantitative time-dependent analysis of the ellipse rotation that takes place whenever the fiber is fed with an amplitude-stabilized field. The more general case of propagation in a single-mode fiber with arbitrary birefringence has been considered in a stationary situation, ${ }^{7}$ which deals with changes in both the ellipticity and the azimuth of the polarization ellipse (for bulk media, see Ref. 8).

The present paper concerns the relevant situation in which the input radiation undergoes amplitude fluctuations. In this case, the polarization ellipse fluctuates as well, so the most significant quantity describing the influence of optical Kerr effect on electromagnetic (e.m.) propagation turns out to be the degree of polarization. In Section 2, we deal with the general formalism, which permits us to investigate the main features of nonlinear coupling between the two circularly polarized states characterizing propagation in a lowbirefringence single-mode fiber and to work out the explicit expression of the field. In Section 3, we obtain the dependence of the degree of polarization on the distance traveled for chaotic radiation (exponentially distributed input intensity). This situation, in addition to being interesting per se, allows us to emphasize the common features of polarization behavior at large distances for an arbitrary initial intensity distribution, as shown in Section 4.

\section{KERR EFFECT AND DEGREE OF POLARIZATION}

A single-mode optical fiber is able to guide two orthogonal linearly polarized states, so the most general guided field is of the form

$$
\begin{aligned}
\mathbf{E}(\mathbf{r}, z, t)= & E_{1}(\mathbf{r}) \exp \left[i \omega_{0} t-i \beta_{1}\left(\omega_{0}\right) z\right] \phi_{1}(z, t) \hat{e}_{x} \\
& +E_{2}(\mathbf{r}) \exp \left[i \omega_{0} t-i \beta_{2}\left(\omega_{0}\right) z\right] \phi_{2}(z, t) \hat{e}_{y},
\end{aligned}
$$

where $E_{1}(\mathbf{r})$ and $E_{2}(\mathbf{r})$ represent the transverse configurations of the two states, $\mathbf{r} \equiv(x, y), \omega_{0}$ is the midfrequency of the field, and $\hat{e}_{x}$ and $\hat{e}_{y}$ are two unit vectors in the $x$ and $y$ directions, respectively. The behavior of the complex amplitudes $\phi_{i}(z, t)$ is connected to source characteristics and coupling between the two orthogonal states, the latter being due to fiber imperfections and to the intensity dependence of the refractive index.

We deal hereafter with a low-birefringence fiber with negligible imperfections and chromatic dispersion, so $E_{1}(\mathbf{r}) \cong$ $E_{2}(\mathbf{r}) \equiv E(\mathbf{r}), \beta_{1} \cong \beta_{2} \equiv \beta$, and the dependence of $\phi_{1}$ and $\phi_{2}$ on $z$ and $t$ is related only to the departure of the source from pure monochromaticity and to the presence of the nonlinear refractive index. More precisely, the evolution of the $\phi_{i}$ is described by the relations ${ }^{6,9}$

$$
\begin{aligned}
& \left(\frac{\partial}{\partial z}+\frac{1}{V} \frac{\partial}{\partial t}\right) \phi_{1}=-i R\left(\left|\phi_{1}\right|^{2}+2 / 3\left|\phi_{2}\right|^{2}\right) \phi_{1}-\frac{i}{3} R \phi^{*}{ }_{1} \phi_{2}{ }^{2}, \\
& \left(\frac{\partial}{\partial z}+\frac{1}{V} \frac{\partial}{\partial t}\right) \phi_{2}=-i R\left(\left|\phi_{2}\right|^{2}+2 / 3\left|\phi_{1}\right|^{2}\right) \phi_{2}-\frac{i}{3} R \phi^{*}{ }_{2} \phi_{1}{ }^{2},
\end{aligned}
$$

where $V=(\mathrm{d} \beta / \mathrm{d} \omega)^{-1}$ (evaluated at $\left.\omega=\omega_{0}\right)$ is the group velocity and 


$$
R=\frac{\omega_{0} n_{2}}{c} \iint_{-\infty}^{+\infty} E^{4}(\mathbf{r}) \mathrm{d} x \mathrm{~d} y \quad\left[\iint_{-\infty}^{+\infty} E^{2}(\mathbf{r}) \mathrm{d} x \mathrm{~d} y=1\right],
$$

$n_{2}$ being the nonlinear refractive-index coefficient, that is,

$$
n=n_{1}+n_{2}|\mathbf{E}|^{2} \text {, }
$$

where $n_{1}$ represents the ordinary linear refractive index of the fiber.

The set of Eqs. (2) can be recast in a simpler form by introducing the circularly polarized states $E(\mathbf{r}) \hat{e}^{+}$(clockwise) and $E(\mathbf{r}) \hat{e}^{-}$(counterclockwise), with

$$
\begin{aligned}
& \hat{e}^{+}=\frac{1}{2^{1 / 2}}\left(\hat{e}_{x}+i \hat{e}_{y}\right), \\
& \hat{e}^{-}=\frac{1}{2^{1 / 2}}\left(\hat{e}_{x}-i \hat{e}_{y}\right) .
\end{aligned}
$$

In fact, if we write the field [see Eq. (1)] as

$$
\mathbf{E}(\mathbf{r}, z, t)=E(\mathbf{r}) \exp \left(i \omega_{0} t-i \beta z\right)\left[\phi^{+}(z, t) \hat{e}^{+}+\phi^{-}(z, t) \hat{e}^{-}\right],
$$

we can easily see that Eqs. (2) are equivalent to

$$
\begin{aligned}
& \left(\frac{\partial}{\partial z}+\frac{1}{V} \frac{\partial}{\partial t}\right) \phi^{+}=-2 / 3 i R\left(\left|\phi^{+}\right|^{2}+2\left|\phi^{-}\right|^{2}\right) \phi^{+}, \\
& \left(\frac{\partial}{\partial z}+\frac{1}{V} \frac{\partial}{\partial t}\right) \phi^{-}=-2 / 3 i R\left(\left|\phi^{-}\right|^{2}+2\left|\dot{\phi}^{+}\right|^{2}\right) \phi^{-} .
\end{aligned}
$$

Equations (7) show that the magnitudes $\left|\phi^{+}\right|$and $\left|\phi^{-}\right|$of the complex amplitudes $\phi^{+}$and $\phi^{-}$, respectively, are constants of motion (see also Ref. 5). In particular, if $\phi^{+}\left(\phi^{-}\right)=$ 0 for $z=0, \phi^{+}\left(\phi^{-}\right)=0$ everywhere. (It is worth noting that, from a fundamental point of view, the above constancy is related to photon-spin conservation, which is, in turn, implied by the symmetry properties of the interaction Hamiltonian corresponding to the Kerr effect.) For a monochromatic source corresponding to $\phi_{0}{ }^{+} \equiv \phi^{+}(z=0)$ and to $\phi_{0}{ }^{-} \equiv$ $\phi^{-}(z=0)$ independent of $t$, the solution of the set of Eqs. (7) read as

$$
\begin{aligned}
\phi^{+}(z) & ={\phi_{0}}^{+} \exp \left[i \gamma^{+}(z)\right], \\
\phi^{-}(z) & ={\phi_{0}}^{-} \exp \left[i \gamma^{-}(z)\right],
\end{aligned}
$$

with

$$
\begin{aligned}
& \gamma^{+}(z)=-2 / 3 R\left(\left|\phi_{0}{ }^{+}\right|^{2}+2\left|\phi_{0}{ }^{-}\right|^{2}\right) z, \\
& \gamma^{-}(z)=-2 / 3 R\left(\left|\phi_{0}{ }^{-}\right|^{2}+2\left|\phi_{0}{ }^{+}\right|^{2}\right) z .
\end{aligned}
$$

In general, i.e., when $\phi^{+}(z=0, t) \equiv \phi_{0}{ }^{+}(t)$ and $\phi^{-}(z=0, t) \equiv$ $\phi_{0}^{-}(t)$ depend on time, Eqs. (7) yield

$$
\begin{aligned}
& \phi^{+}(z, t)=\phi_{0}{ }^{+}(t-z / V) \exp \left[i \gamma^{+}(z, t)\right], \\
& \phi^{-}(z, t)=\phi_{0}{ }^{-}(t-z / V) \exp \left[i \gamma^{-}(z, t)\right],
\end{aligned}
$$

where

$$
\begin{aligned}
& \gamma^{+}(z, t)=-2 / 3 R\left[\left|\phi_{0}{ }^{+}(t-z / V)\right|^{2}+2\left|{\phi_{0}}^{-}(t-z / V)\right|^{2}\right] z, \\
& \gamma^{-}(z, t)=-2 / 3 R\left[\left|\phi_{0}{ }^{-}(t-z / V)\right|^{2}+2\left|\phi_{0}{ }^{+}(t-z / V)\right|^{2}\right] z .
\end{aligned}
$$

Let us now evaluate the degree of polarization $P$, which is, in general, given by the relation ${ }^{10}$

$$
P^{2}=1-\frac{4 \operatorname{det} J}{\left(J_{x x}+J_{y y}\right)^{2}},
$$

with

$$
J=\left|\begin{array}{cc}
J_{x x} & J_{x y} \\
J_{y x} & J_{y y}
\end{array}\right| \equiv\left|\begin{array}{cc}
\left\langle\left|\phi_{1}\right|^{2}\right\rangle & \left\langle\phi_{1} \phi^{*}{ }_{2}\right\rangle \\
\left\langle\phi^{*}{ }_{1} \phi_{2}\right\rangle & \left\langle\left|\phi_{2}\right|^{2}\right\rangle
\end{array}\right|,
$$

where the symbol $(\ldots)$ indicates the ensemble-averaging operation. To this end, we observe that Eqs. (1), (5), and (6) yield

$$
\begin{aligned}
& \phi_{1}=\frac{1}{2^{1 / 2}}\left(\phi^{+}+\phi^{-}\right), \\
& \phi_{2}=\frac{i}{2^{1 / 2}}\left(\phi^{+}-\phi^{-}\right) .
\end{aligned}
$$

After straightforward algebra, Eqs. (10), (11), and (14) permit us to write

$$
\begin{aligned}
2\left|\phi_{1}(z, t)\right|^{2}= & \left|\phi_{10}(t-z / V)\right|^{2}+\left|\phi_{20}(t-z / V)\right|^{2} \\
& +\left[\left|\phi_{10}(t-z / V)\right|^{2}-\left|\phi_{20}(t-z / V)\right|^{2}\right] \cos (\alpha z) \\
& +\left[\phi_{10}(t-z / V) \phi^{*}{ }_{20}(t-z / V)\right. \\
& \left.+\phi^{*}{ }_{10}(t-z / V) \phi_{20}(t-z / V)\right] \sin (\alpha z) \\
2\left|\phi_{2}(z, t)\right|^{2}= & \left|\phi_{10}(t-z / V)\right|^{2}+\left|\phi_{20}(t-z / V)\right|^{2} \\
& -\left[\left|\phi_{10}(t-z / V)\right|^{2}-\left|\phi_{20}(t-z / V)\right|^{2}\right] \cos (\alpha z) \\
& -\left[\phi_{10}(t-z / V) \phi^{*}{ }_{20}(t-z / V)\right. \\
& \left.+\phi^{*}{ }_{10}(t-z / V) \phi_{20}(t-z / V)\right] \sin (\alpha z), \\
2 \phi_{1}(z, t) \phi_{2}^{*}(z, t)= & \phi_{10}(t-z / V) \phi^{*}{ }_{20}(t-z / V) \\
& -\phi^{*}{ }_{10}(t-z / V) \phi_{20}(t-z / V) \\
& +\left[\left|\phi_{20}(t-z / V)\right|^{2}\right. \\
& \left.-\left|\phi_{10}(t-z / V)\right|^{2}\right] \sin (\alpha z) \\
& +\left[\phi_{10}(t-z / V) \phi^{*}{ }_{20}(t-z / V)\right. \\
& \left.+\phi^{*}{ }_{10}(t-z / V) \phi_{20}(t-z / V)\right] \cos (\alpha z),
\end{aligned}
$$

with

$$
\begin{aligned}
i \alpha= & 2 / 3 R\left[\phi_{10}(t-z / V) \phi^{*}{ }_{20}(t-z / V)\right. \\
& \left.-\phi^{*}{ }_{10}(t-z / V) \phi_{20}(t-z / V)\right]
\end{aligned}
$$

$\phi_{10}$ and $\phi_{20}$ refer to the input field $(z=0)$. In principle, we can evaluate the degree of polarization given by Eq. (12) in the most general case by using Eq. (13) and the ensemble averages of Eqs. (15).

\section{DEGREE OF POLARIZATION IN THE CASE OF A CHAOTIC INPUT}

We wish to consider the case of a chaotic input field (exponential intensity distribution), such as the one generated by a light-emitting diode or a multimode laser. ${ }^{11}$ Let us also assume a linear polarization transformed into an elliptical one through a linear optical system placed between the source and the fiber so that the input polarization ellipse 


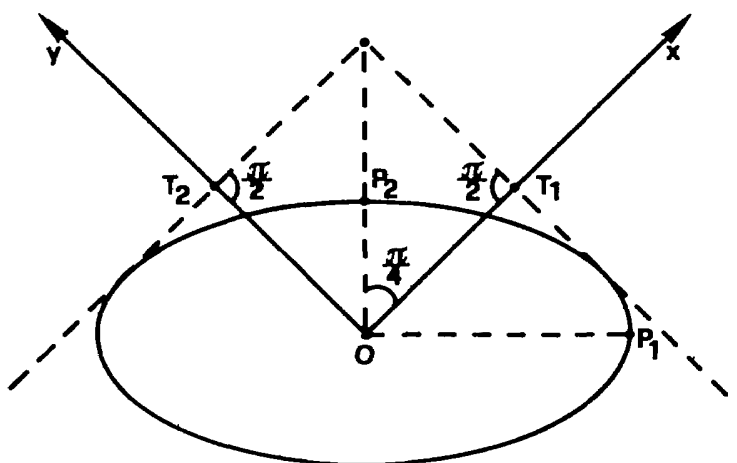

Fig. 1. Input-ellipse geometry: $O P_{1}=A, O P_{2}=B, O T_{1}=\left|\phi_{10}(t)\right|=$ $O T_{2}=\left|\phi_{20}(t)\right|$

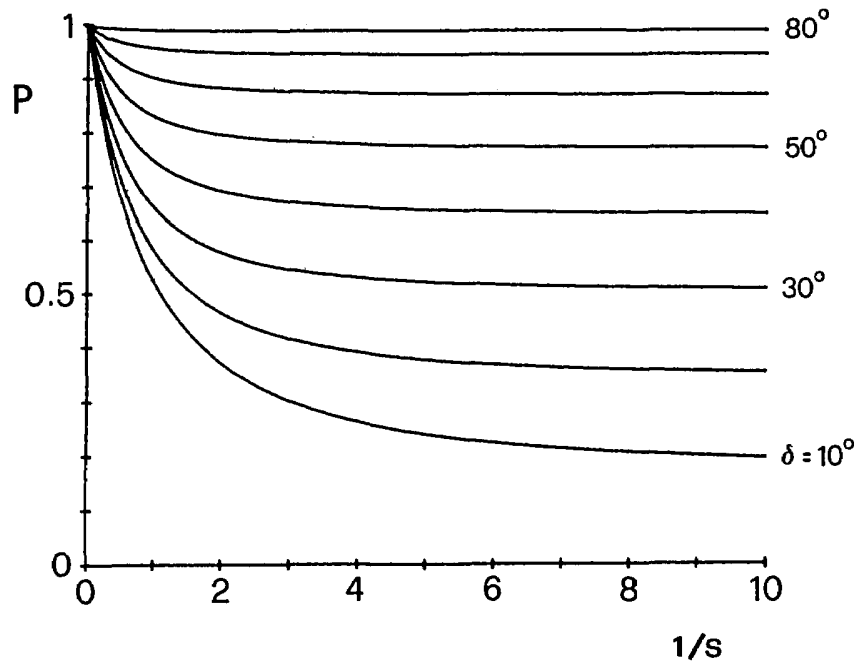

Fig. 2. Degree of polarization $P$ as a function of the parameter $1 / s$ for various values of $\delta$.

fluctuates in magnitude while its ellipticity and orientation remain constant. The above situation corresponds to

$$
\phi_{10}(t)=\exp (i \delta) \phi_{20}(t)
$$

(with $\delta$ time independent), where, for simplicity and without lack of generality, we have chosen the $x$ and $y$ axes forming an angle of $\pi / 4$ with the ellipse axes, which corresponds to equal amplitudes $\left[\left|\phi_{10}(t)\right|=\left|\phi_{20}(t)\right|\right]$. The phase $\delta$ is easily shown to fulfill the relation

$$
|\tan (\delta / 2)|=A / B
$$

where $A$ and $B$ are the amplitudes of the ellipse semiaxes (see Fig. 1).

The assumption of exponential intensity distribution

$$
\begin{aligned}
p\left(\left|\phi_{10}\right|^{2}\right) & =\frac{\exp \left[-\left|\phi_{10}\right|^{2} /\left\langle\left|\phi_{0}\right|^{2}\right\rangle\right]}{\left\langle\left|\phi_{0}\right|^{2}\right\rangle}, \\
p\left(\left|\phi_{20}\right|^{2}\right) & =\frac{\exp \left[-\left|\phi_{20}\right|^{2} /\left\langle\left|\phi_{0}\right|^{2}\right\rangle\right]}{\left\langle\left|\phi_{0}\right|^{2}\right\rangle}
\end{aligned}
$$

allows us to perform [with the help of Eqs. (15) and (17)] the ensemble averages appearing in Eq. (13), so Eq. (12) yields

$$
P^{2}(z, \delta)=1-\left(\cos ^{2} \delta\right) \frac{1+2 s}{1+2 s+s^{2}},
$$

with

$$
s=\frac{9}{16 R^{2} z^{2}\left\langle\left|\phi_{0}\right|^{2}\right\rangle^{2} \sin ^{2} \delta} .
$$

By inspecting Eqs. (20) and (21), it is easy to verify that $P(0, \delta)=1$ (as obviously implied by the fixed orientation of the input ellipse) and that

$$
P(\infty, \delta)=|\sin \delta| \quad(\delta \neq 0, \quad \delta \neq \pi),
$$

while

$P(z, 0)=P(z, \pi)=P(z, \pi / 2)=P(z,-\pi / 2)=1$

(for every $z$ ).

In order to provide an estimate of the decrease undergone by the degree of polarization as a function of the fiber length $z$, we note that [see Eq. (3)]

$$
R=k n_{2} / \sigma \quad\left(k=\omega_{0} / c\right),
$$

where $\sigma$ (effective area of the mode) can be approximated by the core area. ${ }^{3}$ Accordingly, Eq. (21) yields

$$
s=\frac{9 \sigma^{2} n_{1}{ }^{2}}{16 \times 4 k^{2} n_{2}{ }^{2} z^{2} Z_{0}{ }^{2} P^{2}},
$$

where $Z_{0}$ is the vacuum impedance and $P$ is the e.m. power. For typical values of $\sigma\left(\cong \pi a^{2}\right.$, with $\left.a=2.5 \mu \mathrm{m}\right), \lambda(\cong 1 \mu \mathrm{m}), n_{2}$ $\left(\cong 10^{-22} \mathrm{~m}^{2} / \mathrm{V}^{2}\right)$, and $n_{1}(\cong 1.5)$, one has, in MKS units,

$$
s=\frac{2 \times 10^{3}}{(z P)^{2}} \text {. }
$$

Since (see Fig. 2) the polarization decrease becomes observable for $s \leqq 1$ (we remember that $s=\infty$ for $z=0$ ), provided that $\delta$ differs significantly from the relevant values relative to Eq. (23), a fiber length of $\cong 5 \mathrm{~m}$ and an injected power of $\cong 10 \mathrm{~W}$ are sufficient to observe the depolarization effect.

\section{ASYMPTOTIC DEGREE OF POLARIZATION FOR ARBITRARY INPUT-INTENSITY DISTRIBUTION}

In this section, we wish to show that the asymptotic behavior for $z \rightarrow \infty(s \rightarrow 0)$ furnished by Eq. (22) can be obtained for an arbitrary input-intensity distribution. In this respect, we observe that the magnitude of the ellipse rotation undergone at a given $z$ in correspondence to a fixed variation of the input intensity is an increasing function of $z$ itself. Therefore, for (fixed) $z$ large enough, a small change of the initial intensity gives rise to a large number of complete rotations of the polarization ellipse without essentially modifying its amplitude. As a consequence, the asymptotic degree of polarization is conveniently evaluated as the one relative to a field whose ellipse rotates at random with assigned shape and magnitude. To this end, we note that the above field is essentially described by the equation

$$
\begin{aligned}
\mathbf{E}_{\psi}(t)= & \left(\hat{e}_{x} \cos \psi+\hat{e}_{y} \sin \psi\right) E_{x} \cos (\omega t) \\
& +\left(\hat{e}_{y} \cos \psi-\hat{e}_{x} \sin \psi\right) E_{y} \sin (\omega t) \\
= & \hat{e}_{x}\left[E_{x} \cos \psi \cos (\omega t)-E_{y} \sin \psi \sin (\omega t)\right] \\
& +\hat{e}_{y}\left[E_{y} \cos \psi \sin (\omega t)+E_{x} \sin \psi \cos (\omega t)\right],
\end{aligned}
$$


where $\psi$ is the random rotation angle ( $\psi=0$ corresponding to ellipse axes parallel to $\hat{e}_{x}$ and $\hat{e}_{y}$ ).

To evaluate the degree of polarization, ${ }^{10}$ we must, by definition, introduce an arbitrary delay $w$ between the $x$ and $y$ field components in order to evaluate the intensity $I_{\psi}(w, \theta)$ associated with the field projection along an arbitrary direction $\theta$ in the $x-y$ plane and to average $I_{\psi}(w, \theta)$ itself over the random variable $\psi$. The resulting quantity $I(w, \theta)$ allows us to evaluate $P$ through the relation

$$
P=\left(I_{\max }-I_{\min } /\left(I_{\max }+I_{\min }\right),\right.
$$

where $I_{\max }$ and $I_{\min }$ are the maximum and minimum values attained by $I(w, \theta)$ as a function of $w$ and $\theta$. Following the above procedure, we easily obtain from Eq. (27)

$$
\begin{aligned}
I_{\psi}(w, \theta) \propto & \cos ^{2} \theta\left(E_{x}^{2} \cos ^{2} \psi+E_{y}^{2} \sin ^{2} \psi\right) \\
& +\sin ^{2} \theta\left(E_{y}^{2} \cos ^{2} \psi+E_{x}^{2} \sin ^{2} \psi\right) \\
& +2 \cos \theta \sin \theta\left(E_{x} E_{y} \cos ^{2} \psi \sin w\right. \\
& +E_{x}^{2} \sin \psi \cos \psi \cos w \\
& \left.-E_{y}{ }^{2} \sin \psi \cos \psi \cos w+E_{x} E_{y} \sin ^{2} \psi \sin w\right),
\end{aligned}
$$

which yields, averaging over $\psi$,

$$
I(w, \theta) \propto \frac{1}{2}\left(E_{x}{ }^{2}+E_{y}{ }^{2}\right)+2 \cos \theta \sin \theta E_{x} E_{y} \sin w,
$$

so

$$
I_{\max } \propto\left(E_{x}+E_{y}\right)^{2} / 2, \quad I_{\min } \propto\left(E_{x}-E_{y}\right)^{2} / 2,
$$

and [see Eq. (28)]

$$
P=\left(2 E_{x} E_{y}\right) /\left(E_{x}{ }^{2}+E_{y}{ }^{2}\right) .
$$

We now observe that $E_{x}$ and $E_{y}$ represent the ellipse semiaxes, so they coincide with the quantities $A$ and $B$ appearing in Eq. (18) ( $E_{x}=A$ and $E_{y}=B$, or, equivalently, $E_{x}=B$ and $E_{y}=A$ ). Thus comparing expressions (18) and (32) furnishes

$$
P(\infty, \delta)=|\sin \delta| \quad(\delta \neq 0),
$$

where the symbol $\infty$ indicates that we are dealing with large values of $z$.

In conclusion, Eq. (33) indicates that, under our assumption of an input polarization ellipse fluctuating in magnitude but not in ellipticity and orientation [see Eq. (17)], the asymptotic degree of polarization is completely determined by the ellipticity itself and is independent of the intensity distribution of the exciting source. As is obvious, the traveled distance over which the asymptotic value is practically attained depends on the intensity distribution and on the value of $\delta$. In particular, $P$ is equal to unity for all $z$ if the input intensity does not flucutate or (for symmetry reasons) if the input field is linearly or circularly polarized.

It is worth noting that our results hinge on the assumption of negligible birefringence and fiber imperfection. The first hypothesis corresponds to the condition $\left(\beta_{1}-\beta_{2}\right) L \ll 2 \pi$, that is, $L / L_{p} \ll 1$, where $L$ is the length over which a significant ellipse rotation occurs and $L_{p}$ is the fiber beat length, whereas the second hypothesis is equivalent to $L / L_{c} \ll 1, L_{c}$ being a typical distance over which the linear mode coupling associated with fiber imperfection takes place. The first requirement can easily be met by employing a low-birefringence fiber, for which $L_{p}$ can be made of the order of few hundred of meters, ${ }^{12}$ while the second one strongly depends on the environmental conditions under which the fiber is kept. In any case, our analysis, in principle, could be generalized to include birefringence and coupling by relying on the results describing the evolution of the state of polarization of a deterministic field..$^{13,14}$ Finally, we observe that the neglection of group-velocity dispersion underlying our results is justified because of the (relatively) short distances over which significant nonlinear interaction can take place.

\section{ACKNOWLEDGMENT}

This research was partially supported by the Italian Ministry of Education (MPI).

Bruno Crosignani and Paolo Di Porto are also with the Fondazione Ugo Bordoni, Roma, Italy.

\section{REFERENCES AND NOTES}

1. R. H. Stolen, "Nonlinearity in fiber transmission," Proc. IEEE 68, 1232-1236 (1980).

2. R. H. Stolen, "Active fibers," in New Directions in Guided Waves and Coherent Optics, D. B. Ostrowsky and E. Spitz, eds. (Nijhoff, The Hague, 1984), Vol. I, pp. 1-22.

3. R. H. Stolen and C. Lin, "Self-phase modulation in silica optical fibers," Phys. Rev. A 17, 1448-1453 (1978).

4. B. Crosignani, S. Piazzolla, P. Spano, and P. Di Porto, "Direct measurement of the nonlinear phase shift between the orthogonally polarized states of a single-mode fiber," Opt. Lett. 10, 8891 (1985).

5. P. D. Maker, R. W. Terhune, and C. M. Savage, "Intensitydependent changes in the refractive index of liquids," Phys. Rev. Lett. 12, 507-509 (1964).

6. B. Crosignani and P. Di Porto, "Intensity-induced rotation of the polarization ellipse in low-birefringence single-mode optical fibers," Opt. Acta 32, 1251-1258 (1985).

7. B. Daino, G. Gregori, and S. Wabnitz, "Stability analysis of nonlinear coherent coupling," J. Appl. Phys. 58, 4512-4514 (1985); H. G. Winful, "Self-induced polarization changes in birefringent optical fibers," Appl. Phys. Lett. 47, 213-215 (1985).

8. K. L. Sala, "Nonlinear refractive-index phenomena in isotropic media subjected to a dc electric field: exact solutions," Phys. Rev. A 29, 1944-1956 (1984).

9. For a detailed derivation of equations analogous to Eqs. (2) in the case of modes possessing the same linear polarization, see B. Crosignani, A. Cutolo, and P. Di Porto, "Coupled-mode theory of nonlinear propagation in multimode and single-mode fibers: envelope solitons and self-confinement," J. Opt. Soc. Am. 72, 1136-1141 (1982).

10. M. Born and E. Wolf, Principles of Optics VI (Pergamon, Oxford, 1980), p. 552 .

11. M. Bertolotti, B. Crosignani; P. Di Porto, and D. Sette, "Photostatistics of an $N$-mode laser field," Z. Phys. 205, 129136 (1967).

12. S. R. Norman, D. N. Payne, and M. J. Adams, "Fabrication of single-mode fibers exhibiting extremely low polarization birefringence," Electron. Lett. 15, 309-311 (1979).

13. H. G. Winful, "Polarization instabilities in birefringent nonlinear media: application to fiber-optic devices," Opt. Lett. 11, 33-35 (1986).

14. B. Daino, G. Gregori, and S. Wabnitz, "New all-optical devices based on third-order nonlinearity of birefringent fibers," Opt. Lett. 11, 42-44 (1986). 